Mots. Les langages du politique

$83 \mid 2007$

Dire la démocratie aujourd'hui

\title{
Oui ou non. Le piège rhétorique du référendum
}

Bertrand Pirat

\section{CpenEdition}

Journals

Édition électronique

URL : https://journals.openedition.org/mots/967

DOI : $10.4000 /$ mots.967

ISSN : 1960-6001

\section{Éditeur}

ENS Éditions

\section{Édition imprimée}

Date de publication : 1 mars 2007

Pagination : 139-153

ISBN : 978-2-84788-111-0

ISSN : 0243-6450

\section{Référence électronique}

Bertrand Pirat, "Oui ou non. Le piège rhétorique du référendum », Mots. Les langages du politique [En ligne], 83 | 2007, mis en ligne le 01 mars 2009, consulté le 22 avril 2022. URL : http:// journals.openedition.org/mots/967; DOI : https://doi.org/10.4000/mots.967

\section{(c) ENS Éditions}




\section{Bertrand Pirat}

\section{Oui ou non. Le piège rhétorique du référendum}

L'article 2 du décret du 9 mars 2005 décidant de soumettre au référendum le projet de loi autorisant la ratification du Traité constitutionnel européen (TCE), est formulé de la manière suivante :

Les électeurs auront à répondre par «oui » ou par «non» à la question suivante : «Approuvez-vous le projet de loi qui autorise la ratification du traité établissant une Constitution pourl'Europe?»

La question référendaire et ses modalités de réponse attendues constituent un contexte d'énonciation spécifique : oui et non sont utilisés comme adverbes équivalant à une proposition, laquelle répond à une interrogation. Dans ce contexte d'énonciation, oui et non sont des pro-phrases, strictement équivalentes à une proposition, affirmative ou négative ${ }^{1}$. Le commentaire qui précède la question organise donc le choix démocratique par une symétrie des réponses, entre « répondre par oui » et « [répondre] par non ». Cette symétrie est le gage de l'impartialité et de la neutralité civique du dispositif de consultation populaire. Conformément à l'idéal démocratique, il suppose que chaque citoyen, après avoir jugé chaque disposition du Traité constitutionnel, puisse évaluer l'ensemble du texte sur la base de ces jugements et se prononcer par «oui » ou par «non ». Les deux mots-phrases sont alors le produit d'un processus de réflexion individuel et d'émergence d'une opinion, d'une part, et d'autre part le moyen de réponse prévu, sur la base de cette opinion, selon un usage normé des mots oui et non, mots "vides », équivalents elliptiques de la réponse à la question posée, soit au positif, soit au négatif.

Ce face-à-face solitaire de chaque citoyen avec la question référendaire ne correspond qu'à une partie de la réalité du processus de consultation populaire, tant du point de vue des phénomènes politiques qu'il génère que du point de vue des faits de langue qui se manifestent à cette occasion. Les médiations entre le citoyen et la question du référendum sont multiples. Elles sont parties prenantes du sens du vote «oui» ou du vote «non». Les médias nationaux

1. On transpose ici au contexte référendaire le questionnement d'ordre pragmatique initié par Christian Plantin à propos de oui et non en contexte dialogique (voir Plantin, 1982, p. 252-265).

Université Lumière-Lyon 2, Institut d’études politiques de Lyon, bertrand.pirat@univ-lyon2.fr 
constituent une première médiation entre la question référendaire et les citoyens. S'ils contribuent massivement à constituer et à faire partager un cadrage du référendum, ce n'est pas seulement en produisant un flux d'actualité générale sur lequel vient prendre place le traitement médiatique de celui-ci². Les dispositifs médiatiques de discussion publique, tels que le comptage du temps de parole, la composition des invités et leur disposition, ainsi que les jeux de langage qui leur sont associés, comme les commentaires des sondages d'opinion ou l'étiquetage des acteurs politiques, tendent à consolider, en référence à la fois au dispositif référendaire et aux usages civiques d'égalité devant l'épreuve du suffrage, la symétrie instituée par les pro-phrases « oui » et « non ».

Les forces politiques constituent une seconde médiation entre le citoyen et la question référendaire. Quelles que soient les motivations imputées au président de la République quant au choix du référendum³ ${ }^{3}$ la forme de la consultation référendaire constitue une contrainte forte pour les organisations politiques. Le caractère dialogique du référendum implique indissociablement une réponse à la question posée et à celui qui la pose. La question fermée oblige au choix dual pour exprimer publiquement une prise de position sur l'objet du référendum. Ainsi les organisations partisanes, mais aussi les organisations connexes au champ politique, syndicales et protestataires, sont contraintes de « prendre parti » pour le « oui » ou pour le « non ». Mais à l'inverse des citoyens cantonnés à l'usage de leur bulletin de vote, les porte-parole des organisations politiques ont la possibilité de produire le sens qu'ils entendent attribuer à leur parti pris référendaire, à travers une prise de position qui excède la simple reprise de la pro-phrase. Loin de constituer seulement des mots «vides », oui et non génèrent alors des faits de langue propres au contexte d'énonciation de la campagne. Oui et non sont l'objet d'une intense bataille de construction de sens, dont l'enjeu est d'imposer une interprétation spécifiquement politique aux réponses référendaires. Ce sont deux entités sémantiques à politiser, à partir d'un sens commun déjà là qui fonctionne potentiellement comme contrainte ou comme ressource rhétorique, par la mise en œuvre des ethos ${ }^{4}$ qui leur sont attachés.

La multitude des formules, consignes, mots d'ordre, slogans, qui sont produits et énoncés durant la campagne référendaire en utilisant les mots oui et/ou non, constitue un site d'observation privilégié de la construction du sens des

2. Sur cet aspect de la réalité médiatique, voir Piar, Gerstlé, 2005, p. 42-73.

3. Ainsi le choix de Jacques Chirac pour la solution référendaire peut être analysé a posteriori comme motivé par des considérations de politique intérieure : contraindre les leaders de son propre camp à le soutenir, diviser l'opposition de gauche. Mais l'examen du contexte national et international antérieur à la décision fait apparaitre son caractère contraint (voir Laurent et Sauger éd., 2005).

4. Ce travail tente d'illustrer l'articulation possible entre la nouvelle rhétorique de Chaim Perelman et la sociologie des champs de Pierre Bourdieu autour de la notion commune d'ethos, comprise dans sa double dimension discursive et sociale (sur cette question théorique, voir Amossy, 1999, p. 127-154). 
votes. Par slogan, on entend ici très classiquement « une formule concise et frappante, facilement répétable, polémique [...] et destinée à faire agir » (Reboul, 1975, p. 42). Le propre du slogan efficace est d'être repris bien au-delà de son auteur et de se confondre avec l'évènement politique en obligeant chacun des acteurs à prendre position par rapport à ce qu'il énonce. Force est de constater que la campagne relative à la ratification du TCE n'a pas donné lieu à l'émergence d'un slogan hégémonique pour chaque réponse référendaire mais, au contraire, à la multiplication des formules constituées à partir des mots oui et/ ou non, qui peuvent être considérés comme des candidats slogans. Le corpus ${ }^{5}$ a été constitué selon deux modalités différentes. Un recueil de tracts distribués sur les marchés lyonnais du $7^{\mathrm{e}}$ arrondissement a donné accès à des campagnes de communication nationales, mais aussi à des documents issus de structures politiques départementales ou locales (annonce de meeting, appel à manifester, etc.). Une recherche sur Internet a permis de compléter le corpus afin de couvrir plus largement le spectre politique français. L'ensemble ainsi constitué ne prétend cependant pas à l'exhaustivité, ni même à une représentativité de la totalité des slogans produits pendant la campagne. Il vise en revanche à rendre compte de tous les effets d'ethos permis par l'usage de oui et de non dans ce contexte, en fonction d'une position d'énonciation dans le champ politique. Chaque slogan a été associé à son instance d'énonciation et, lorsque c'était nécessaire à la compréhension, aux éléments sémiotiques d'inscription qui l'accompagnent, comme la mise en page, la typographie ou d'éventuels logos.

Le contexte dialogique propre au référendum institue donc un usage normé de oui et de non à travers une symétrie syntaxique entre les pro-phrases. Le rappel de ce contexte de référence permet d'apprécier la plus ou moins grande proximité sémantique entre la question référendaire et les usages des deux adverbes mis en slogan dans la campagne. L'analyse du corpus en langue montre une symétrie des formes grammaticales utilisées dans ces mises en slogan et dans la nature des actes de langage qu'elles autorisent. Une analyse en discours montre en revanche une dissymétrie des effets de sens produits par l'usage de oui et de non. La mise en slogan des réponses référendaires contribue à imposer le sens politique des votes par la mobilisation ou la neutralisation des ethos attachés à oui et à non tels qu'ils apparaissent dans les stratégies discursives des différents énonciateurs, en fonction de leur position dans le champ politique. Cela aboutit à créer une asymétrie symbolique entre les deux réponses ${ }^{6}$.

5. Je tiens à remercier Marie-Claude Dunand, responsable du service de documentation de l'Institut d'études politiques de Lyon, pour son aide lors de la constitution de ce corpus.

6. Je remercie Marlène Coulomb-Gully de m'avoir permis d'exposer une version antérieure de ce travail lors de la journée organisée par la Société d'étude des langages du politique (SELP) à Toulouse, le 21 octobre 2005. Je remercie également Pierre Fiala pour ses suggestions lors de cette journée. 


\section{Symétrie en langue}

\section{Oui et non comme adverbes}

La construction grammaticale utilisée dans la question référendaire est celle d'un adverbe équivalant à une proposition et répondant à une question. Cette construction grammaticale se retrouve, dans les slogans de campagne, surtout sous forme de logo. Ainsi, un « oui ! » peut se lire en vignette sur un flyer invitant à une manifestation pro-« oui » en surplomb du slogan «Faites l'Europe! ». De même pour un «non », figurant en lettres capitales avec la mention "Une autre Europe est possible » à l'intérieur d'un 0 constitué par les étoiles de la bannière européenne. Oui et non sont, dans ce type de contextes, des réponses qui renvoient implicitement à la question référendaire. Elles ne peuvent se comprendre qu'en rapport avec cette question. Leur usage dans la mise en slogan est symétrique, tant d'un point de vue pragmatique que grammatical. La nature adverbiale de oui et de non est donc la plus proche de la question référendaire. Elle reste cependant marginale dans le corpus.

\section{Oui et non comme compléments directs}

Une deuxième structure grammaticale place oui et non en position de complément direct d'un verbe déclaratif. Cette structure grammaticale offre, là encore, une parfaite symétrie entre les deux modalités de réponse à la question référendaire et dans la mise en slogan qu'elles suscitent: il y a symétrie entre « voter oui » et «voter non », entre les formes de l'impératif «votez oui / non», ou du démonstratif déclaratif «je vote oui / non», entre leur formes elliptiques respectives «oui à » et «non à », et entre les infinitifs « dire oui » et «dire non». Cette dernière forme rend possibles de nombreuses variantes selon la nature du complément d'objet choisi. Ainsi, dire « oui » / «non » permet de préciser un substantif («oui » / «non » à quelque chose) susceptible d'être qualifié, mais aussi dire «oui » / «non » avec quelqu'un susceptible d'une appartenance ou d'un ralliement:

Avec les socialistes de toute l'Europe, dites OUI au traité constitutionnel. (Tract national, PS)

À travers les usages dont elles sont susceptibles d'être l'objet dans les slogans, les structures grammaticales de oui et de non, comme compléments directs d'un verbe déclaratif, contribuent, par la symétrie syntaxique, à la neutralité du dispositif référendaire. Elles rendent également explicite la dimension performative du vote à venir: la propriété originale de ces «oui » et de ces «non» 
est d'acquérir force de loi, en fonction de la règle de la majorité. Au performatif s'ajoutent les effets de métonymie du dispositif référendaire et de ses jeux de langage, qui construisent le groupe de ceux qui votent oui et celui de ceux qui votent non. C'est la forme nominale de oui et de non qui réalise cette métonymie.

\section{Oui et non comme noms}

Une troisième structure grammaticale permet la mise en slogan : oui et non comme nom masculin invariable, noyau d'une phrase nominale. «Un oui » et « un non » autorisent chacun à qualifier la réponse à la question référendaire par diverses expansions et positionnent cette réponse comme un moyen en vue d'une fin : «un oui pour », « un non pour». Ainsi « un oui » comme « un non » sont susceptibles d'être qualifiés par un adjectif ou une forme adjectivée qui contribue à leur attribuer un sens :

Un NON d'avenir !

(Tract, Appel des 200)

Un OUI d'avenir!

(Site Internet : lesamisduoui.com ; éditorial de Jean-François Legaret, premier viceprésident du groupe UMP du Conseil de Paris, maire du $1^{\text {er }}$ arrondissement)

La forme nominale précédée de l'article indéfini renvoie encore à la dimension performative du vote et à la métaphore vocale de l'opinion : voter, c'est s'exprimer, se prononcer, faire entendre sa voix. Cette construction syntaxique tend à transformer l'opinion énoncée ou mobilisée des «oui » et des «non» exprimés durant la campagne référendaire en opinions discrètes potentiellement dénombrables et agglomérables. Le non et le oui deviennent alors des agrégats qui s'affrontent dans un rapport quantitatif, largement utilisé par les commentateurs de la vie politique et de l'évolution de l'opinion publique sondée, et dont le rapport se fige dans l'épreuve référendaire.

Les formes grammaticales de oui et de non encouragent donc à penser les unités référendaires "oui » et "non » comme deux entités symétriques. Utilisés en tant qu'adverbes avec statut de pro-phrases, comme compléments directs d'un verbe déclaratif ou comme noms, oui et non autorisent une mise en slogan selon des structures syntaxiques symétriques et vont aboutir en discours à des faits de langue mimétiques : tous deux sont, potentiellement et tout à la fois, une réponse à une question, un acte performatif, une opinion exprimée, une "voix» proclamée; un " oui » et un " non » sont également des suffrages potentiellement exprimés et comptabilisés le jour du scrutin, «le oui » et «le non » sont les deux partis qui s'opposent dans la cité référendaire.

Mais la mise en slogan ne répond pas uniquement aux contraintes syntaxiques ou pragmatiques de la langue. Elle révèle des usages qui répondent à des 
logiques agonistiques, propres au contexte d'énonciation du discours politique. Les unités linguistiques oui et non s'inscrivent dans des faits de langue plus riches que les usages normés par les structures grammaticales ou par le dispositif référendaire. Elles font l'objet de construction de sens et de stratégies discursives différenciées, où se jouent à la fois l'émergence d'un parti pris dans le débat public, l'élaboration de son image, la reconstruction de l'image de l'adversaire politique et la réponse à sa propre image imposée par l'adversaire. L'analyse du corpus en discours, c'est-à-dire en fonction des contraintes pragmatiques liées au contexte socio-politique, montre au contraire une dissymétrie des usages politiques de oui et de non, liée au poids symbolique que représente accord ou opposition dans le champ politique.

\section{Dissymétrie en discours}

Une analyse de contenu fondée sur une décomposition des unités du corpus en syntagmes indépendants de la forme slogan montre une dissymétrie entre l'usage de oui et l'usage de non, dans la construction de sens que chacun rend possible. Pour obtenir une mise à plat du corpus qui soit significative et pour éviter les artefacts qu'aurait occasionnés une analyse systématique indépendante du contexte pragmatique, le tri des syntagmes doit prendre en compte les modalités de mise en équivalence logique (alignement ou contre-alignement) entre le sens du vote mis en slogan, et le lien d'équivalence logique établi avec le syntagme, indépendamment du mot réponse utilisé dans le slogan. En effet, les partisans du « oui » utilisent parfois le mot non dans leur slogan - et réciproquement. Un classement en quatre catégories permet d'obtenir une présentation synoptique des syntagmes mobilisés par chaque camp et de caractériser leur modalité d'usage.

Le « oui » comme le «non » définissent positivement l'Europe en alignant les substantifs :

Pour un oui de gauche à l'Europe du 29 mai.

(Tract local, Comité pour le oui du $3^{\mathrm{e}}$ arrondissement)

Je rêve d'une véritable Europe sociale, laïque et républicaine. Au nom de cette belle

Europe, je voterai NON au référendum sur ce Traité

(Comité du Rhône pour le non socialiste, PRS [Pour une République sociale])

Le «non » peut également définir négativement l’Europe par contre-alignement:

Un NON d'avenir!

L'Europe du OUI à la Constitution, c'est celle des Chirac, Schröder, Blair, Berlusconi contre lesquels se sont mis en grève Français, Italiens, Allemands, Hollandais et bien d'autres en 2004. (Tract local, Appel des 200) 
Mais le «non » consacre une majorité de syntagmes à qualifier la Constitution par contre-alignement :

\author{
Votez NON à cette « Constitution européenne » \\ Non à cette Constitution libérale ! \\ Non à cette Constitution antidémocratique ! \\ Non à cette constitution militariste et atlantiste ! \\ (Trac local, Appel des 200)
}

Le «oui », enfin, ne suscite que très peu de contre-alignements, dont voici un des rares exemples:

Je refuse la simple zone de libre échange.

Je refuse de rester sur un grand marché.

Je refuse le statu quo.

$[\ldots]$

Je vote oui.

(La lettre de l'Europe par Martine Rourre, députée européenne, éditorial, PS et PSE)

Cet élément de dissymétrie rejoint et confirme un constat intuitif, inscrit dans les usages sédimentés de la langue et les structures de la conversation ordinaire : dire « oui » est synonyme d'acquiescer mais aussi d'accepter et d'admettre. Oui est actif. Dire «non » signifie aussi nier, refuser, repousser7. Non est réactif. II est donc logique que le camp du « oui » contre-aligne peu et préfère utiliser le mot oui, aligné sur des valeurs positives comme l'Europe ${ }^{8}$. Parce qu'elle mobilise la métaphore qui associe le vote à la performance vocale, parce qu'elle actualise la dimension performative de l'expression de l'opinion, la mise en slogan des réponses référendaires nécessite la prise en compte du sens produit par l'usage des mots oui et non, qui joue potentiellement comme contrainte ou comme ressource pour celui qui l'énonce. Pour rendre compte de la dissymétrie sémantique entre oui et non, il faut prendre en compte la situation d'énonciation propre au slogan. Par destination, le slogan est comme une parole sans locuteur, engagée tout entière vers sa réception9. II n’en produit pas moins des

7. Le Robert, 1993.

8. L'analyse de conversation montre que dans les situations qui présentent une alternative, une seule des deux réponses est dite "préférée», valant comme participation et comme engagement dans la situation: "[...] assessments may be seen as products of participation in social activities, the proffering of them is part and parcel of participating in such activities. That is, they are occasionned conversationnal events with sequential contraints, where on major locus of their occurences is on the occasions of the participation » (Pomerantz, 1984, p. 58).

9. Cette caractéristique autorise Dominique Maingueneau à inclure le slogan parmi les phénomènes relevant du discours rapporté et d'un « régime de participation » de l'énonciation : «Le slogan, à l'instar du proverbe, ne peut qu'être répétable. Le slogan se désigne comme doublement répétable : il se répète d'un lieu de participation [...] à l'autre. Il est en outre indéfiniment répété par ceux qui le scandent. Il implique un ethos approprié : en l'occurrence un ethos qui marque un engagement de toute la personne. [...] L'énonciation du slogan militant implique l'existence d'un extérieur hostile ou indifférent par rapport auquel se pose le groupe » (Maingueneau, 2004, p. 119). 
effets d'ethos qui s'exercent non dans la projection d'une image de l'orateur dans son propos ${ }^{10}$, mais par la production d'un ethos d'identification ${ }^{11}$ qu'il faut chercher dans ce que l'énoncé suggère lui-même de son énonciation. Anticipant la réception, la mise en slogan d'une prise de position politique implique nécessairement une maîtrise - toujours relative - de cet ethos d'identification. L'usage obligé des mots oui et non pour prendre position dans le débat public fonctionne comme une ressource ou comme une contrainte dans la construction de l'ethos d'identification.

\section{Oui comme ressource}

L'usage de oui offre une coalescence potentielle entre ses différentes formes grammaticales. Les trois formes grammaticales de oui peuvent se surajouter et se compléter dans un même slogan. Même dans un format court, oui se prête facilement à la sloganisation :

\section{L'Europe a besoin de notre OUI}

OUI, dans un monde de plus en plus dominé par la puissance américaine et bientôt chinoise, il nous faut une Europe plus forte.

OUI, pour que les peuples s'y retrouvent, l'Europe doit devenir plus démocratique.

Donnons la parole aux citoyens et reconnaissons leurs droits sociaux.

OUI, il faut une Constitution pour l'Europe.

[...]

Nous sommes engagés.

Nous votons OUI.

(UDF, tract national)

À la forme nominale qui désigne l'orientation du vote vient s'ajouter le « oui » détaché devant une phrase affirmative pour annoncer ou souligner celle-ci, et enfin le performatif « Nous votons OUI » incluant la pro-phrase elliptique, renforcé par l'assertion « Nous sommes engagés » qui explicite l'ethos actif.

D'autre part, oui tend à se suffire à lui-même : on trouve ainsi des «oui! » en logo, ou simplement renforcés par un adverbe - «Franchement oui !» (PS, tract national) ; «Tout simplement oui ! (UDF, annonce du meeting de François Bayrou à Lyon) - et par l'adjonction du point d'exclamation. S'il peut se suffire à lui même, c'est parce que oui concentre une réponse référendaire, une forme

10. On n'ignore pas que cette campagne a donné lieu à un usage personnalisé des slogans et à des enjeux intra-partisans. Mais c'est la relation entre instance politique et instance citoyenne qui constitue notre objet. Pour une discussion de la notion d'ethos en analyse du discours politique, voir Simone Bonnafous, «La question de l'ethos et du genre en communication politique », http://www.cerimes.fr/colloquefrancomexicain/actes/ethos.htm, site consulté le 27 décembre 2005.

11. "Toute construction d'ethos se fait dans un rapport triangulaire entre soi, l'autre et un tiers absent porteur d'une image idéale de référence : le soi cherche à endosser cette image idéale, l'autre se laisse emporter par un mouvement d'adhésion à la personne qui s'adresse à lui par l'intermédiaire de cette même image idéale de référence. » (Charaudeau, 2005, p. 105) 
grammaticale autonome (le mot-phrase) et une catégorie logique de mise en équivalence : oui =. Utiliser oui précédé d'un adverbe permet, avec une économie de moyens, de renvoyer à des traits de caractères associés à l'ethos actif: ici, franchise et simplicité.

Cet ethos actif de oui est mobilisé lorsque la réponse référendaire est utilisée dans des contextes renvoyant à des situations qui font appel à des qualités humaines (courage, volonté, droiture). Ainsi, l'ethos actif se manifeste en réponse à une circonstance difficile :

L'Europe a besoin de notre OUI.

(UDF, tract national)

Ou encore, en réponse à une situation de jugement: dire « oui », par analogie avec le grand «oui » (du mariage), relève ici de la pure volonté, du choix raisonné en conscience, délibéré :

L'Europe mérite qu'on lui dise oui.

(UMP, site Internet)

\section{Oui comme contrainte}

Le caractère univoque et monolithique de l'ethos actif associé à oui constitue en revanche une contrainte pour ceux qui ont à se démarquer politiquement de l'initiateur du référendum afin d'exprimer leur position politique sur l'objet de la consultation. Ainsi le slogan du Parti socialiste traduit le caractère contraignant du dualisme référendaire :

L'Europe sociale passe par le oui.

(PS, tract national)

La métaphore utilisée ("passer par») associe le référendum à une étape de la construction européenne et fait du vote «oui » un point de passage obligé pour parvenir à une fin : l'Europe sociale. La conflictualité potentielle du clivage «oui »/«non», superposé au clivage Europe sociale / Europe libérale, s'en trouve totalement désamorcée. L'ethos actif de oui est euphémisé par la métaphore du détour.

Ce « oui » sous contrainte du Parti socialiste ne manque pas d'être exploité par les forces politiques qui se positionnent à gauche et pour le «non ». L'ethos actif de oui est toujours susceptible d'être disqualifié par excès de positivité :

Voter oui, c'est accepter l'Europe du chômage !

(Tract départemental, appel des 200 , collectif du Rhône)

Le cheminement argumentatif est un syllogisme qui mobilise l'ethos actif de oui : si voter «oui » est synonyme de dire « oui » et dire «oui » synonyme 
d'accepter, alors voter «oui » c'est accepter, notamment l'inacceptable. Ces équivalences sémantiques mobilisent également les stéréotypes sociaux associés à l'énonciation de oui. En filigrane de cette disqualification de l'ethos actif de oui, se trouve la figure du béni-oui-oui ${ }^{12}$, qui articule le mot oui, des mœurs oratoires et une posture publique.

\section{Non comme ressource}

La mise en slogan du vote «non» produit un effet d'ethos réactif. Cet ethos d'identification et de réaction à l'affirmation du choix du oui mobilise en premier lieu les significations liées à l'usage du mot non dans le langage ordinaire. Certains éléments du corpus le font avec une grande économie de moyens, à travers des formules où non est lexicalisé par le présentatif «c'est » :

Pour moi c'est NON.

(Jean-Luc Mélenchon, PRS [Pour une République sociale])

Cette fois-ci, c'est non!

(Collectifs socialistes pour le « non », Henri Emmanuelli, site Internet)

Selon la position occupée par les producteurs du slogan dans le champ politique, cet ethos est mobilisé et amplifié par le contexte sémantique ou désamorcé. Pour les forces politiques dont le positionnement est associé à la contestation de l'ordre social, la campagne référendaire est l'occasion de tirer un profit symbolique de la force réactive de la réponse référendaire négative, et de redonner ainsi une pertinence dans le débat public à une posture de protestation :

Faisons entendre la voix du NON!

(PT, tract départemental, Fédération du Rhône)

L'ethos réactif est ici dans l'énonciation (par la ponctuation) mais également dans l'énoncé : évoquer une «voix du non », c'est souligner la corporéité de la parole politique; affirmer la nécessité pour elle de se faire entendre implique une position dominée et une volonté de renverser le rapport de domination.

L'ethos réactif se retrouve également dans la thématique de la dignité. Sans être nécessairement associé à une posture de contestation radicale, le vote «non» admet le « oui à l’Europe» mais se justifie par une lutte pour la reconnaissance :

[...] je voterai Non au référendum sur ce Traité.

Parce qu'il n'y a pas de fatalité !

12. «Personne toujours empressée à approuver les initiatives d'une autorité établie » (Le Robert, 1996). 
Parce que nous sommes debout!

(Tract départemental, texte en gras, PRS [Pour une République sociale], comité du Rhône pour le non socialiste)

Le «non » est également une ressource lorsque l'adversaire est stigmatisé par la qualification négative de son ethos. C'est le cas lorsque le parti du «oui» (dis)qualifie le «non». Ainsi le collectif du « oui » olympique fait le lien entre le résultat du référendum et la candidature de la France aux Jeux olympiques de 2012 et assortit son argumentaire d'un logo : «non sens ». L'argument suggéré porte ici sur la disqualification de l'ethos réactif associé à non et utilisé par le camp du «non ».

\section{Non comme contrainte}

Dans le cadre d'un usage politique des mots, ce qui est ressource pour les uns peut se révéler contrainte pour les autres. C'est justement le cas pour tous ceux qui, obligés d'en passer par ce mot pour exprimer leur position sur la construction européenne, tentent de contenir la force réactive du refus que comporte le sens de $n o n^{13}$, pour qu'il n'aboutisse pas en définitive à la mise en équivalence des clivages «oui »/«non», pour l'Europe / contre l'Europe, le vote «non» équivalant alors à une position disqualifiée au sein du champ politique ${ }^{14}$. L'usage d'une forme slogan strictement symétrique à « Oui à l'Europe » étant politiquement impossible, la mise en slogan du «non » donne lieu à un énorme travail pour en désamorcer l'ethos réactif. Toutes les formes slogan peuvent être utilisées dans cette perspective. La forme nominale se prête à un qualificatif positif : «Un NON d'avenir!» (Tract du collectif Appel des 200) ; elle peut aussi être associée à «pour»: «Un NON de gauche pour changer l'Europe!» (Collectif du Rhône, appel des 200, tract local et départemental) ; la forme complément d'objet est neutralisée par l'usage de pour: «Pour construire l'Europe sociale, votons NON à la Constitution libérale » (Tract du collectif du Rhône pour un Non de gauche, antilibéral et européen) ; la forme elliptique est également associée à pour: « Pour l'Europe sociale, non à la Constitution!» (Collectif du Rhône, appel des 200, tract local et départemental). Dans les énoncés qui précèdent se joue explicitement ou implicitement le clivage social/libéral qui a structuré le débat public. Quand un terme du clivage est formulé, cela suffit à impliquer l'autre. Cet usage contradictoire de pour associé à non a pour objectif de contrer l'équivalence logique, le figement syntagmatique et le positionnement politique : NON = CONTRE. L'ethos réactif de non se lit également à travers

13. Sur le «non » comme acte de langage fondateur d'un engagement politique, voir « Dire non en politique », Mots. Les langages du politique, $1995, \mathrm{n}^{\circ} 45$.

14. Cette disqualification est explicitée par Jacques Chirac lors de sa dernière allocution télévisée avant le référendum, le 26 mai 2005. Il déclare: «On ne peut pas se revendiquer européen et dire "non".» 
le travail de dénégation dont il est le moteur, par l'usage délibérément paradoxal et provocateur d'un sentiment positif: "J'aime l'Europe, je vote non!» (Nicolas Dupont-Aignan, UMP, président des clubs « Debout la République »), comme une inversion du « oui » du mariage. Une dernière modalité de neutralisation de l'ethos réactif de non réside dans sa juxtaposition avec oui :

\section{Oui à l’Europe}

Non à cette Constitution

(Logo, site Internet, Laurent Fabius)

Ce slogan déjoue, en l'explicitant, le piège rhétorique potentiellement contenu dans la question référendaire: si voter «oui» c'est autoriser la ratification du Traité établissant une Constitution pour l'Europe, voter «non » peut signifier voter contre l'Europe. Pour mettre en slogan la position du «non proeuropéen », il est nécessaire de circonscrire l'ethos réactif au seul texte constitutionnel.

Si la forme dialogique de la consultation référendaire tend à instituer une symétrie des réponses en «oui » et «non » sur le modèle des pro-phrases, si les slogans de la campagne référendaire sont constitués, pour le « oui » comme pour le «non », à partir des formes grammaticales identiques, l'analyse du contexte sémantique de chaque slogan montre comment se constitue un ethos d'identification propre à oui et à non. Selon la prise de position, la localisation dans le clivage droite/gauche, la construction d'un ethos actif ou réactif, à partir des mots oui ou non, est accentuée ou au contraire neutralisée.

\section{Asymétrie symbolique}

Une lecture des effets d'ethos dans la mise en slogan des réponses référendaires nécessite cependant la prise en compte plus large du contexte d'énonciation. La spécificité du contexte référendaire réside dans la pratique, par de multiples acteurs, de jeux de langage qui font exister «le oui » et «le non», formes nominales, comme les deux partis de la cité référendaire. La mise en slogan équivaut alors à un travail politicien de partition de la cité et de construction d'une appartenance au parti du «oui » ou au parti du «non». Le parti du « oui » et le parti du «non » acquièrent une modalité d'existence plus prégnante encore lorsque les discours des protagonistes intègrent, dans une sorte de réflexivité, cette modalité d'énonciation de «oui » et de «non», par une auto-qualification politicienne :

Les ouvriers, les employés, les salariés et retraités, les privés d'emploi, les jeunes, les peuples d'Europe, les femmes et les hommes de gauche ont tout à gagner avec le «NON » au référendum. Sa victoire sera votre victoire. (Tract national, PCF) 
Mais l'usage de la forme nominale porte en elle-même une dynamique de montée aux extrêmes lorsque chaque position est reformulée par ses adversaires. Ainsi les partisans du « oui » utilisent le mot non pour qualifier le parti adverse, et réciproquement :

L'Europe du OUI à la Constitution, c'est celle des Chirac, Schröder, Blair, Berlusconi, contre lesquels se sont mis en grève Français, Italiens, Allemands, Hollandais et bien d'autres en 2004.

Votez NON à cette «Constitution européenne »

(Tract, appel des 200)

Le « oui » et le « non » ne sont pas seulement des partis qui s'opposent dans la cité référendaire, ils peuvent également se présenter comme des forces engagées dans une relation agonistique. ${ }^{15}$ Le contexte d'énonciation est constitué par ce rapport quantitatif qui instaure nécessairement une différence qualitative. Une asymétrie symbolique se constitue par la dynamique du rapport entre forces actives et forces réactives:

Le doute taraude le oui.

Le mécontentement s'enfle.

Dites NON à la Constitution des patrons!

(Appel à un meeting d'union du non, LCR)

Si la force du «oui » est mise en cause, c'est à partir d'une position réputée dominante dans un rapport de force. Lorsque le rapport de force s'inverse, l'ethos de non ne devient pas actif, mais son caractère réactif est disqualifiant:

Si le «NON» l'emporte, tout projet de Constitution européenne sera abandonné. (Tract national, en gras, UDF)

Le vote «non » menace ici non seulement l'existence du projet de Constitution européenne soumis au référendum mais également la possibilité de «tout projet » de Constitution européenne, c'est-à-dire tous les projets à venir. C'est la puissance de négation de non qui disqualifie le vote «non ». L'ethos réactif est disqualifié par son excès.

La dynamique de la campagne référendaire transfigure les réponses en deux camps opposés qui s'affrontent, à travers une bataille de clivages, pour imposer le sens du «oui » et le sens du «non » à travers des usages de oui et de non.

15. Le concept nietzschéen de force est particulièrement pertinent pour rendre compte de la métaphysique politique implicite qu'expriment les jeux de langage référendaires: "Les forces ont une quantité, mais elles ont aussi la qualité qui correspond à leur différence de quantité : actif et réactif sont les qualités des forces. [...] La qualité d'une force n'est pas autre chose que la différence de quantité, et qui correspond dans chaque force en rapport. » (Deleuze, 1994, p. 48, 49 et 50) 
Mais ces luttes symboliques sur le sens des positions politiques sont aussi des luttes d'images de soi, où les acteurs s'entre-définissent à travers leur ethos et celui qu'ils renvoient de leur adversaire. Ethos actif et ethos réactif ne peuvent cependant correspondre au strict affrontement symétrique d'un camp contre un autre, tel que le suggère la forme de la question référendaire. Leur qualification mutuelle dans le jeu de langage du rapport de force ne peut que creuser l'écart symbolique qui s'établit entre le « oui » et le «non». Donnée gagnante dans les sondages d'opinion avant le début de la campagne, soutenue par le chef de l'État, les leaders des partis dits « de gouvernement » et les médias centraux, la réponse « oui » a revêtu un caractère quasi officiel ${ }^{16}$, contribuant ainsi au cadrage antérieur des significations du «non » face au «oui », et donnant en définitive au «non» réactif sa dimension polémique. Le «non » français au référendum sur le Traité constitutionnel européen constitue, du fait même de cette asymétrie symbolique, un évènement discursif dont les effets de sens continuent de se construire a posteriori. Réduire sa signification à un refus de l'Europe serait ignorer la multiplicité des prises de position qui se sont exprimées à partir de la réponse majoritaire, et laisser se refermer le piège rhétorique du référendum.

\section{Références}

AMOSSY Ruth, 1999, "L'ethos au carrefour des disciplines. Rhétorique, pragmatique, sociologie des champs», R. Amossyéd., Image de soi dans le discours. La construction de l'ethos, Lausanne, Paris, Delachaux et Niestlé.

CHARAUDEAU Patrick, 2005, Le discours politique. Les masques du pouvoir, Paris, Vuibert.

DELEUZE Gilles, 1994, Nietzsche et la philosophie, Paris, Presses universitaires de France.

LAURENT Annie et SAUGER Nicolas éd., 2005, Les Cahiers du CEVIPOF, Le référendum de ratification du Traité constitutionnel européen : comprendre le «Non» français, $\mathrm{n}^{\circ} 42$.

MAINGUENEAU Dominique, 2004, "Hyperénonciateur et "participation" », Langages, $\mathrm{n}^{\circ} 156$.

PIAR Christophe, GERSTLÉ Jacques, 2005, « Le cadrage du référendum sur la Constitution européenne : la dynamique d'une campagne à rebondissements », Les Cahiers du CEVIPOF, supra.

PLANTIN Christian, 1982, "Oui et non sont-ils des “pro-phrases” ? Remarques sur leur fonctionnement dans les dialogues », Le Français moderne, $n^{\circ} 50$ (3), juillet.

16. On retrouve le même type d'asymétrie entre les notables locaux et les notabilités locales d'État bénéficiant des attributs matériels et symboliques de la candidature officielle dans la France du $19^{\mathrm{e}}$ siècle (voir Voilliot, 2005). 
POMERANTZ Anita, 1984, "Agreeing and disagreeing with assessments : some features of preferred / dispreferred turn shapes », J. M. Atkinson et J. Heritage éd., Structures of Social Action: Studies in Conversation Analysis, Cambridge, Cambridge University Press.

REBOUL Olivier, 1975, Le slogan, Paris, Complexe.

VOILLIOT Christophe, 2005, La candidature officielle. Une pratique d'État de la Restauration à la Troisième République, Rennes, Presses universitaires de Rennes (Carnot). 